\title{
Marco Bianchi Il dire galileiano per titoli
}

\section{Una nota lessicale su /l Saggiatore*}

\begin{abstract}
The importance of Galileo's use of language is well known. He also paid particular attention to the titling of his works. This paper shows that the only correct acceptation of the title Il Saggiatore (1623) is 'the man who assays' and not 'assay balance', as generally interpreted (the ambiguity, too, is not possible). We reach this conclusion on the basis of different elements, such as the discussion of the historical usage of the word, the historical semantics of the suffix -tore and contemporary interpretations. Furthermore the paper explains the stylistic potential of that title and its conceptual allusions.
\end{abstract}

Keywords: Galileo Galilei, suffix -tore, title, science and literature

Galileo Galilei, suffisso -tore, titolo, scienza e letteratura

Marco Bianchi: Ruprecht-Karls-Universität Heidelberg, Romanisches Seminar, Seminarstr. 3, 69117 Heidelberg, E-Mail: marco.bianchi@rose.uni-heidelberg.de

\section{II precedente del Sidereus nuncius}

Tra il 1609 e il 1610 Galileo vide con il cannocchiale ciò che nessun uomo aveva visto. Affidò il racconto delle osservazioni a una breve opera latina, stesa e stampata in fretta, che egli stesso sapeva destinata a procurargli la fama in tutta Europa (e ancor più lontano): il Sidereus nuncius. 'Messaggero' o 'messaggio'?' Senza dubbio Galileo lo intese nel significato di 'notizia astronomica', così da presentare il testo quale neutro (ma sconvolgente) bollettino di avvisi. «Mando all'Altezza Vostra Ser.ma il mio Avviso Astronomico, dedicato al suo felicissimo nome» (EN 10, 297): così scrive il 19 marzo 1610 inviando al Granduca di Toscana

\footnotetext{
* Ringrazio coloro che hanno letto versioni precedenti di questo articolo e mi hanno stimolato con le loro osservazioni e obiezioni: Andrea Battistini, Michele Camerota, Daniela Goldin, Pier Vincenzo Mengaldo, Lorenzo Renzi.

1 Puntate della querelle sull'accezione del titolo sono Rosen (1950), Rosen (1957), Drake (1958), Russo (1967); cf. le considerazioni di Pantin (1992, XXXII-XXXVII) e di Battistini (1993, 177s.).
} 
una copia dell'opera. ${ }^{2}$ Quando negli anni successivi indicherà in italiano il titolo dell'opera, dirà per lo più avviso astronomico oppure nunzio sidereo (cf. Drake 1958, 347). Circa quest'ultimo sintagma, avendo nunzio la medesima ambiguità del latino ed essendo per giunta il sintagma italiano una mera trasposizione dell'originale, non ha senso volerne dedurre una genuina accezione del titolo, come vorrebbe Drake. Come glossa di Galileo su se stesso non resta che avviso astronomico, del resto pienamente corrispondente all'ideologia, alla struttura e allo stile dell'opera. Avviso astronomico è anche il titolo scelto da Viviani, ultimo discepolo e collaboratore dello scienziato, per la sua traduzione italiana. ${ }^{3}$

Tutto ciò non toglie, tuttavia, che l'autore fosse consapevole dell'ambiguità che il lettore avrebbe colto in una parola tanto comune come nuntius (qualora avesse voluto disambiguare, aveva a disposizione il neutro nuntium, meno frequente del maschile, ma pur sempre possibile). La stessa correzione dell'ultim'ora, quando il libro era in parte già stampato, da Astronomicus nuncius, come si legge ancora nella titolazione che segue la dedicatoria, a Sidereus nuncius, contribuì ad accrescere il possibile equivoco. ${ }^{4}$ La modifica, oltre a collegarsi direttamente ai medicea sidera scoperti dallo scienziato, era in primo luogo dovuta a un movente di pulizia etimologica, in base al quale un sintagma mezzo greco (astronomicus) e mezzo latino (nuncius) venne sostituito con un altro tutto di stampo latino (sidereus nuncius). Ma vi era anche la volontà di de-tecnicizzare il titolo: astronomicus sembrava infatti suggerire che il discorso si svolgesse solo all'interno della disciplina (e l'astronomia, considerata allora branca della matematica, era ricca di «pure ipotesi» cui si negava realtà fisica), mentre sidereus, richiamandosi semplicemente ai corpi celesti (sidera), lasciava da parte le sovrastrutture disciplinari e suggeriva un'aderenza alla realtà fisica degli oggetti osservati. Con un ordine certo intenzionale, il frontespizio precisava che l'opera era rivolta «philosophis atque astronomis» ('ai filosofi naturali e agli astronomi'), assai più significativo perché Galileo era correttamente indicato solo con la qualifica di mathematicus, qual era presso lo Studio di Padova. Parimenti, però, l'aggettivo sidereus rese possibile, nell'ambiguità di nuncius, il significato di 'messaggero celeste', di cui Galileo dovette essere consapevole e che in seguito

2 Esplicita, ma in un contesto dominato dalla polemica, è anche una postilla al Grassi: «Io non mi son mai chiamato ambasciador sidereo; nè voi per tale mi areste nominato, se aveste inteso il titolo del mio libro, il quale è inscritto Sidereus Nuncius, che vuole dire Ambasciata o Avviso Sidereo, e non Ambasciadore» (EN 6, 388s.). Tale nota è posteriore al 1626, anno di pubblicazione della Ratio ponderum di Grassi.

3 Firenze, Biblioteca Nazionale Centrale, Ms. Gal. 48, cc. 46-53; cf. Favaro (1892).

4 In due documenti dei Riformatori dello Studio di Padova si trova una terza titolazione, Astronomica denuntiatio ad astrologos (EN 19, 227s.; cf. Pantin 1992, 55s.). 
accettò, tra gli altri, da parte di un lettore importante come Kepler, che seppe giocare retoricamente con quel titolo nella sua Dissertatio cum Nuncio sidereo. ${ }^{5}$

\section{Il caso del Saggiatore: motivazioni lessicali per l'accezione 'colui che saggia'}

A differenza di Sidereus nuncius, il titolo Il Saggiatore (1623) non ebbe nella sua epoca alcuna ambiguità: significava senz'altro, come credo di poter dimostrare, 'colui che saggia' e non 'strumento con cui si saggia, bilancia da saggiatori', come si è detto in genere. A partire da Anton Maria Salvini sino al GDLI, infatti, si è chiosato il titolo sottolineandone la polisemia e risolvendosi per lo più per l'accezione strumentale. ${ }^{6}$ È pur vero che un passo del primo capitolo del Saggiatore può trarre in inganno. Così scrive Galileo rivolgendosi al destinatario Cesarini:

Ho nondimeno mantenuta l'istessa risoluzione di parlar con V. S. Illustrissima ed a lei scrivere, qualunque si sia poi riuscita la forma di questa mia risposta; la quale ho voluta intitolare col nome di Saggiatore, trattenendomi dentro la medesima metafora presa dal Sarsi. Ma perché m'è paruto che, nel ponderare egli le proposizioni del signor Guiducci, si sia servito d'una stadera un poco troppo grossa, io ho voluto servirmi d'una bilancia da saggiatori, che sono così esatte che tirano a meno d'un sessantesimo di grano: e con questa usando ogni diligenza possibile, non tralasciando proposizione alcuna prodotta da quello, farò di tutte i lor saggi; i quali anderò per numero distinguendo e notando, acciò, se mai fussero dal Sarsi veduti e gli venisse volontà di rispondere, ei possa tanto più agevolmente farlo, senza lasciare indietro cosa veruna (EN 6, 220).

5 All'esempio di Viviani (Avviso astronomico) dovrebbero conformarsi, a mio parere, le traduzioni straniere, tra le quali alcune (The starry messenger, The sidereal messenger, Le messager celeste, Le messager des étoiles) presentano come centrale quello che è in realtà solo un significato possibile giocosamente alluso (il gioco della retorica).

6 Salvini chiosa il titolo galileiano in una nota alle commedie di Buonarroti il Giovane (Buonarroti 1726, 391). Vi sono naturalmente eccezioni: Besomi/Helbing $(2005,474)$ illustrano la genuina accezione allegando una postilla galileiana alla Ratio ponderum (la seconda di quelle che citerò); Reusch $(1879,201)$ allinea in nota alcune delle testimonianze che espongo qui, senza trarne però la giusta conclusione, giacché a testo afferma che il titolo «man könnte 'die Goldwage' übersetzen». Saggiatore viene per lo più tradotto in inglese assayer, in francese essayeur, in tedesco Goldwa(a)ge. Drake (1995, 284) afferma che l'opera galileiana «was called Il Saggiatore, meaning 'the assayer', in allusion to the fine balance used for weighing gold as against the ordinary balance implied by Grassi's title, Libra». Chauvire $(1979,39)$ dedica una ricca nota al titolo e alla sua traduzione, affermando che «on pourrait [...] traduire «Saggiatore» par 'Trébuchet’ pour mettre en relief la symétrie entre le livre de Galilée et la Balance de Sarsi [...] Nous avons néanmoins préféré conserver le terme 'essayeur', plus conforme à une tradition déjà bien établie dans les études galiléennes». Entrambi gli studiosi abbracciano in sostanza la tesi della polisemia del termine. 
Per i motivi linguistici di diverso ordine che sono allineati e discussi qui di seguito, ritengo che l'affermazione di essersi tenuto con quel titolo «dentro la medesima metafora presa dal Sarsi» vada riferita al campo semantico generale del pesare e del saggiare, e non implichi che saggiatore sia uno strumento qual è la libra. Con il tempo, proprio questo accostamento e lo sviluppo del suffisso -tore in accezione strumentale hanno contribuito a un'errata interpretazione del titolo.

a. L’uso galileiano. Nel corpus galieiano il termine non designa mai uno strumento, ma sempre la persona. Titolo a parte, le due occorrenze della parola nel Saggiatore stesso sono comprese nel sintagma «bilancia da saggiatori»; similmente nel Dialogo, dove Sagredo propone: «Ma sentiamo il rimanente delle ragioni favorevoli alla sua opinione, per venir poi al lor cimento, coppellandole e ponderandole con la bilancia del saggiatore» (EN 7, 157). Tre postille dello scienziato alla Ratio ponderum di Orazio Grassi sono esplicite:

Voi [si rivolge direttamente all'avversario] non intendete questo mestiero, mentre che voi credete che i saggiatori si servino delle bilancine per pesar l'oro o l'argento, essendo che l'uffizio è di ritrovare se 'l metallo che vien proposto per oro puro o per argento, è tale, o pure tiene di rame o altra materia men perfetta, o è alchimia etc.: e così il Saggiatore scuopre i vostri errori mascherati con molte molte fraudi e 'nganni, e non gli pesa altramente, lasciando che tal giudizio si faccia da chi si sia e con qualsivoglia stadera ben grossa (EN 6, 382). ${ }^{7}$

Se voi aveste cognizione della lingua toscana, aresti, senza più oltre leggere nel mio libro, inteso come il nome Saggiatore senza traslazione significa l'istesso che collibista, e non quello che praegustator vini, il quale noi chiameremmo assaggiatore, poi [che] si dice assaggiare il vino, e non saggiare (EN 6, 381). ${ }^{8}$

Voi di sopra, per darmi, con arguzia assai fredda, del bevitore e briaco, dite che, sendo la prima origine di questa parola Saggiatore presa dall'assaggiare i vini, onde saggiuoli etc., fuste per esprimerla con il termine pitissator, libator, etc.; ma che poi avendo dalla lettura del mio libro compreso che io la pigliavo per significar quelli che fanno i saggi dell'oro, parendovi anco che la prima denominazione fusse poco onesta e indegna di filosofo, e che per ciò ne desideravi una più sobria, lasciata la prima, pigliaste quest’altra (EN 6, 386).

b. L'interpretazione dei contemporanei. Non sono a conoscenza di contemporanei di Galileo che abbiano inteso il titolo altrimenti che come nomen agentis. In

7 Il commento di Galileo si riferisce a questo passo della Ratio ponderum: «Fausta hinc ominari libet Librae meae, cuius si qua sunt peccata, exigua adeo censentur, ut eorum ponderi libra grandior non succumbat, quaeque non nisi minutissima simbella, quam vel atomus presset, examinari possint» (EN 6, 382).

8 La parentesi quadra è nell’EN. 
appendice al suo Tychonis Brahei Dani Hyperaspistes (1625), Kepler inserisce l'appendice Spicilegium ex Trutinatore Galilaei, dove appunto il titolo Saggiatore è reso con Trutinator; per quanto Kepler non conoscesse l'italiano, è evidente che gli era stato suggerito quel significato. ${ }^{9}$ Grassi e il traduttore latino del Saggiatore che agì per impulso di Galileo - probabilmente Marco Ambrogetti - lo traducono simbellator. ${ }^{10}$ Entrambe le rese evitano l'ambiguità, giacché distinte dai rispettivi strumenti trutina e simbella. Nella Ratio ponderum Librae et Simbellae Grassi ammette la difficoltà di tradurre in latino quel titolo:

ab ipso operis nomine exordiamur; cui latinitati donando haud sane parum mihi laborandum fuit, ut nesciam an quicquam aliud, in toto hoc volumine confutando, mihi molestius acciderit. Quamquam enim permultae occurrebant voces, e latinitatis foecundissimo penu depromptae, idem re ipsa sonantes, iis tamen hetruscae vocis sapor minus exprimi videbatur (EN 6, 380).

La difficoltà è in parte reale, in parte sarcastica: sapor allude al mestiere del sommelier («qui vina degustat, libat, pitissat»), che Grassi presenta come valore originario di saggiatore, attirandosi le dure critiche della seconda postilla galileiana sopra riportata. Per la traduzione, Grassi si è infine deciso per simbellator: «malui ab ipsa simbella, novo quamvis vocabulo, Simbellatorem dicere» (EN 6, 382). Nell'antichità simbella (o sembella) si trova solo in Varrone per indicare una moneta del valore di mezza libella (l'etimo è appunto SEMI-LIBELLA); pur assente, se non per questo richiamo erudito, nei lessici del latino medievale attualmente disponibili, la parola dovette assumere nel tempo anche l'accezione di 'bilancia', come testimonia il Thesaurus dello Stephanus (1531), che, oltre all'accezione antica, riporta il significato di «instrumentum parvum quo singuli nummi ponderantur». ${ }^{11}$ In italiano, troviamo simbella in una lettera di Francesco Rinuccini a Galileo (EN 18, 258): «Mi par mill'anni di veder queste sue cose su la simbella di V. S. Ecc.ma» (6 ottobre 1640).

c. Attestazioni lessicali. A parte il caso del titolo galileiano, non è attestato nei lessici (Crusca, Tommaseo/Bellini, GDLI, GRADIT, DELI) né nel corpus LIZ un caso di saggiatore 'strumento' che sia anteriore al 1667. Il corpus TLIO presenta

9 Lo Specilegium è raccolto in Kepler (1963, 413-425; cf. anche il Nachbericht in fondo al volume).

10 La traduzione latina dell'opera è contenuta nel Ms. Gal. 316 della Biblioteca Nazionale Centrale di Firenze, cc. 59-149. La traduzione è oggetto della mia tesi di dottorato (in corso).

11 Ė dunque da escludere l'etimologia proposta da Favaro e Del Lungo, che non avevano peraltro rintracciato altre occorrenze del termine: da сүмвula (сумвеLla), сумва 'piccola barca', che ondeggia come la bilancia (Del Lungo/Favaro 1911, 309). 
occorrenze tutte agentive; ${ }^{12}$ il medesimo risultato ha dato una consultazione, puramente indicativa, di Google Books. Nei Saggi di naturali esperienze (1667) si ha la prima attestazione strumentale: «sulle bilance dette il saggiatore» (256), unica occorrenza del lemma nell'opera, ${ }^{13}$ mentre nel diario dell'Accademia del Cimento, alla data 12 luglio 1657, si legge: «su le bilance del saggiatore». ${ }^{14}$ Tramite Magalotti il lemma entra nella terza Crusca $(1691)^{15}$ e di lì si diffonde, soprattutto nell'Ottocento, in altri lessici e nella lingua colta, come dimostrano nomi di riviste e di case editrici. Non è sicuro, però, che abbia avuto un uso tecnico nell'ambiente della saggiatura dei metalli. ${ }^{16}$ Il Rigutini/Fanfani registra solo l'accezione agentiva, come pure Il tesoro della lingua italiana. Vocabolario nomenclatore di Palmiro Premoli (1909-12, s. v. saggio e bilancia). Pur nell'incompletezza dei repertori lessicali, mi pare plausibile che il passaggio semantico dalla persona allo strumento, che pure è avvenuto, almeno nella lingua alta, debba non poco all'omonimo scritto galileiano; anzi, probabilmente esso fu determinante. Ciò sembra confermato dall'assenza di un analogo sviluppo semantico di ensayador e essayeur in spagnolo e francese (nonché nell'inglese assayer): ${ }^{17}$ all'estero l'influenza culturale di quest'opera è stata assai minore (prova indiretta ne è che si sono avute traduzioni integrali solo in anni recentissimi: la prima nel 1960 ad opera di Stillman Drake). ${ }^{18}$

d. Diacronia lessicale del suffisso - tore. Il duplice valore agentivo e strumentale del suffisso -tore è comune nell'italiano contemporaneo. Non così in diacronia:

12 Consultato il 16 giugno 2013.

13 Già Poggi Salani $(1961,54)$ segnalava che questa è la prima attestazione di cui disponiamo. La stampa dell'opera fu, come è noto, molto accurata; tuttavia sarebbe da verificare che la lezione «bilance DETTE il saggiatore» non sia un refuso per «bilancETTE Del saggiatore (sintagma attestato peraltro nel Bartoli).

14 Targioni Tozzetti $(1780,618)$.

15 La prima citazione galileiana per il lemma si ha con la quarta edizione (1729-1738), ma non è il titolo del Saggiatore.

16 Lascia anzi qualche sospetto che nell'amplissima trattazione (cinquanta pagine circa) che Francesco Griselini e Marco Fassadoni dedicano all'arte del saggiatore nel quattordicesimo tomo del loro Dizionario delle arti e de' mestieri (1773, s. v. saggiatore, arte del), il termine indichi la persona e non la bilancia. La cosa non può giustificarsi solo con la collocazione veneta dell'opera, visto che anche Guid'Antonio Zanetti nella Nuova raccolta delle monete e zecche d'Italia (1775, vol. 1), riferisce la parola solo a esseri umani. Nell'Ottocento l'accezione strumentale sembra accettata: il Nuovo dizionario universale tecnologico o di arti e mestieri e della economia industriale e commerciante (1833, vol. 11, s. v. Saggiatore o saggiuolo), afferma che è una bilancia e che «si chiamò saggiatore per l'uso cui serve».

17 Non utili al caso specifico Lüdtke (2005) e d'Angelis (2006).

18 Drake/O’Malley (1960). 
nel fiorentino duecentesco pare fosse soltanto agentivo (Bisetto 2010, § 41.2.1.2). Inoltre, è stato mostrato come strumentali (prevalenti o esclusivi) di parole in -(s) ore, forma allomorfa, compaiano a partire dal Cinquecento e diventino via via più frequenti. ${ }^{19}$ Possiamo aggiungere, incrociando i dati di DELI, LEI e GRADIT, che tra i derivati in -tore che secondo quest'ultimo comparvero nel Seicento, solo pulitore e succiatore ebbero in quell'epoca accezione strumentale (sono entrambi attestati in testi tecnici). Due casi in forse sono calcatore e rettificatore: il primo per l'origine del suffisso, da ricondurre a -torium secondo il LEI; il secondo per l'impossibilità di verificare una fonte non più disponibile (cf. DELI e Tommaseo/ Bellini). Gli altri numerosi neologismi, circa 200, o ebbero e hanno solo significato agentivo (miratore, irritatore ecc.) o acquisirono in seguito quello strumentale (alcuni in tempi recentissimi, come puntatore, soffiatore, modulatore). Un ruolo particolare sembrano aver avuto nomi anatomici quali (muscolo) abduttore, adduttore, erettore, otturatore, pronatore, supinatore, tutti attestati proprio a partire dal XVII secolo, che stanno in una posizione intermedia tra agentivo e strumentale, in quanto riferiti a parti di esseri animati. Nel Seicento -tore fu molto produttivo e Galileo stesso, con scopi diversi, coniò almeno crivellatore, legatore, oblatrato$r e .{ }^{20} \mathrm{Ma}$ l'accezione strumentale rimase quasi del tutto inespressa. Sembra strano, peraltro, che un toscano così attento e sensibile ai problemi di lingua qual era Galileo non abbia giustificato l'innovazione lessicale, qualora avesse voluto intendere saggiatore come 'bilancia'. Resta, infine, un problema: «quando il derivato ha il tratto [- umano] si tratta per lo più di un termine tecnico-scientifico (o eventualmente parascientifico), nato dalla cancellazione del $\mathrm{N}$ 'apparecchio, strumento, congegno, macchina, prodotto, sostanza' ecc.». ${ }^{21}$ Dardano si riferisce all'italiano contemporaneo, ma è un'osservazione valida anche per il passato. Così si spiegano abduttore, adduttore e altri. Con saggiatore l'operazione è problematica, perché non ho rintracciato casi di *strumento saggiatore o simili (l'improduttività o quasi di -trice in quel secolo impedisce di pensare a ${ }^{\star}$ bilancia saggiatrice: la forza del suffisso inizia a rivelarsi corposamente nel secondo Ottocento ed esplode nel secolo seguente). Il sintagma più frequente è bilancia da/del saggiatore, e non consta che dal tipo martello da carrozziere, metro da sarto, morsetto da falegname siano derivati un sarto 'metro da sarto' e simili. In più, dal punto di vista semantico, è incerto se saggiatore avrebbe avuto la stessa congruità di termini quali ventilatore, soffiatore, calcolatore: mentre essi riescono infatti a compiere interamente e in automonia l'operazione che potrebbe fare un agente

19 Lo Duca (2004, 369). Sul suffisso -tore cf. anche: Tekavčić (1980, §§ 1483-1489), Dardano (1978), Scalise (1996), Patruno (2005), Passarotti (2007, 85-92), Scalise/Bisetto (2008, 188-193).

20 Non rilegatore, come per errore dice il DELI.

21 Dardano (1978, 52; cf. anche 51 e 57). 
umano, un 'apparecchio saggiatore' non avrebbe allora potuto veramente saggiare, ma soltanto pesare.

e. Altri nomi. Per designare la bilancia da saggiatore erano disponibili altre parole. Nello stesso campo semantico ed etimologico di saggiatore, almeno dal Trecento esisteva saggiuolo: «dicesi anche SAGGIUOLO a quelle bilancette, con che si pesano i fiorin dell'oro» (Crusca 1612). Sforza Pallavicino utilizzerà questo termine in senso figurato: "Ancorché Aristotile nella rettorica paia ciò affermare, tuttavia quivi egli non pesa le sue diffinizioni col saggiuolo della più accurata filosofia»; «In così fatte composizioni possonsi lisciar i periodi al torno e bilanciar i contrapposti nel saggiuolo» (cito dal GDLI, s. v. saggiuolo). Segno che nel 1644, anche per un estimatore di Galileo quale il Pallavicino, era saggiuolo e non saggiatore il termine per la bilancia da saggiatore. Ma a Galileo non dovette piacere, forse per il richiamo all'accezione 'campione di vino o di olio' (GDLI), presente anche nella fase successiva della polemica con Grassi (cf. postille alla Ratio ponderum, EN 6, 381 e 386).

Era possibile usare saggio nell'accezione di ‘bilancia da saggio', come attesta il GDLI (s. v., § 17). Lo stesso padre Riccardi si servì di questo termine nel parere censorio che permise l'imprimatur: egli espresse retoricamente la gioia retorica di vivere al tempo dell'autore, «quando non più con la stadera ed alla grossa, ma con saggi sì delicati si bilancia l'oro della verità», dimostrando di aver colto appieno il gioco linguistico di Galileo. ${ }^{22}$

Vi erano poi bilancina/o e bilancetta, già cinquecenteschi. Galileo si servì del primo termine in una delle postille alla Ratio ponderum citate sopra; il secondo è assente dal corpus galileiano, benché proprio Bilancetta sia il titolo vulgato di un'opera giovanile, testimoniato da uno solo dei manoscritti e impostosi con la stampa bolognese del 1656. ${ }^{23}$ Tale titolo è probabilmente spurio - non ci aiuta in questo l'autografo, anepigrafo -, giacché i testimoni hanno titolazioni differenti tra loro e all'interno del testo Galileo utilizza sempre e solo «bilancia». Non inutile al nostro discorso è rilevare come la copia di questa operetta per mano di Vincenzo Viviani sia intitolata Fabbrica et uso di un'esatta bilancia da saggiatore per ritrovare [...]: ancora una volta saggiatore non indica lo strumento.

22 La lezione «saggi sì delicati» è correzione autografa di Galileo sull'esemplare Bonelli (cf. Besomi/Helbing 2005, 649). Nella princeps era stampato «saggiuoli sì delicati».

23 Non so dire quale fosse il titolo nella princeps, compresa in Hodierna (1644). 


\section{Stile e valore di un titolo}

Per replicare alla Libra e contrastarla fin dal titolo, Galileo avrebbe potuto chiamare la sua opera La bilancia (generico), L'esatta bilancia (per suggerire che quella di Grassi era mal tarata), La bilancetta, La bilancina, La bilancia da saggiatore, e in molti modi ancora (Sarseide ecc.). Non lo fece. La finissima sensibilità sua e dei Lincei - non sappiamo esattamente a chi attribuire la paternità del titolo - dovette escludere tali ipotesi, tutte a vario titolo deboli, specialmente bilancina e bilancetta, che pur appropriate lessicalmente possedevano, causa il diminutivo, poca forza icastica. Fu certo chiarissimo a Galileo che quell'opera brillante cui affidava la trattazione esplicita, per quanto non esauriente, del suo metodo e della sua filosofia e in cui attaccava con ferocia intellettuale Brahe e la scienza dei Gesuiti, dovesse colpire l'immaginario del pubblico colto e affrontare vittoriosa il confronto con la Libra già nel titolo. Del resto, Galileo fu sempre attento ai problemi della titolazione e pienamente consapevole del potenziale comunicativo insito in essa. Lo dimostrano, a diverso grado, il referenziale Sidereus nuncius, che apre nuovi mondi con l'asciuttezza e l'agilità dei commentari di Cesare; l'Istoria e dimostrazioni intorno alle macchie solari, in cui Galileo volle inserire, contro il parere del principe Cesi, la concretezza della sua terminologia e del suo approccio; il censurato titolo Dialogo del flusso e del reflusso del mare, che in una sorta di sineddoche concettuale nominava un'opera tanto vasta e ricca - quella che si intitolò poi Dialogo sopra i due massimi sistemi del mondo tolemaico e copernicano - con l'argomento ritenuto decisivo, che il lettore avrebbe così indelebilmente ricordato (e indelebilmente sarebbe risaltato, nell'immaginario dei posteri, l'errore del grande scienziato: in questo la censura gli fu di vantaggio). Fu del tutto giustificata la scontentezza di Galileo per il titolo che gli Elzeviri diedero alle speculazioni sul moto e sulla meccanica (Discorsi $e$ dimostrazioni intorno a due nuove scienze attinenti alla mecanica e i movimenti locali), che gli parve troppo colloquiale quanto a discorsi - avrebbe probabilmente preferito dialoghi, che sentiva più elevato stilisticamente - e impreciso riguardo alla definizione delle due scienze. Egli ebbe una predilezione per i titoli misti, composti di un elemento meta-linguistico (Lettera, Discorso, Dialogo ecc.) e di un elemento che espone il contenuto tematico (intorno alle macchie solari, del flusso e del reflusso del mare ecc.). ${ }^{24}$

24 Cf. Genette (1987, 54-97); Rothe (1986, in particolare 55-60, 79-84, 180s.); Migliorini (1994, 404; sui titoli secenteschi). Per un quadro contemporaneo sui titoli utilizzati in alcune discipline (anche umanistiche) cf. Dietz (1995). 
Saggiatore fu l'eccezione più vistosa a questo comportamento. Senza dubbio un titolo geniale: fonicamente e graficamente corposo, esso si mantenne nel campo metaforico della Libra, spostando però l'accento dal pesare al saggiare, dal peso alla purità delle affermazioni discusse, e nel contempo - senza dubbio lo slittamento più importante - dallo strumento all'addetto, anzi precisamente dallo strumento a colui che valuta la realtà e la giudica servendosi di uno strumento (la «bilancia esquisita e giusta» del frontespizio). Questa l'opposizione polemica presente nel titolo. È pur vero che nel capitolo introduttivo tale scarto non è sviluppato, perché anche il Grassi usa uno strumento, la sua libra, che però è inadeguato e viene presentato malignamente da Galileo come una stadera, cosicché l'opposizione che domina l'opera è quella tra la bilancia alla grossa (la stadera appunto) e la bilancia di precisione (la bilancia da saggiatori). ${ }^{25} \mathrm{La}$ malignità di presentare la Libra di Grassi non come bilancia, ma come stadera, risale a Giovanni Battista Stelluti: quella che nel frontespizio della Libra (Grassi 1619) era disegnata come bilancia a due piatti, nel frontespizio dello Scandaglio sopra la Libra (Stelluti 1622) diviene una stadera sulla quale una mano applica uno strumento per verificarne l'attendibilità. ${ }^{26}$ Peccato che nel frontespizio definitivo del Saggiatore non sia sviluppata questa iconografia; sappiamo però che un progetto precedente, poi scartato, prevedeva «una figura [...] che habbia conformità con quel titolo di Saggiatore» (EN 13, 121) e che Galileo fornì un disegno a

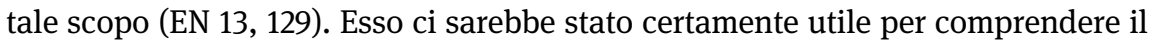
significato del titolo. ${ }^{27}$ Nel capitolo introduttivo del Saggiatore (EN 6, 220) si conferma lo scarto tra il pesare e il saggiare suggerito dal titolo: Grassi pondera le tesi dell'avversario («nel ponderare egli le proposizioni del signor Guiducci»), mentre Galileo dice che saggerà le affermazioni di Grassi («farò di tutte i lor saggi»). Anche una postilla alla Ratio insiste su questo: «il Saggiatore scuopre i vostri errori mascherati con molte molte fraudi e 'nganni» (EN 6, 381). Galileo si riferisce alla verifica della buona e cattiva moneta delle opinioni cometarie, ma anche dei fenomeni naturali che di volta in volta vengono esaminati insieme alle opinioni. Tale identificazione dello scienziato con un saggiatore, pur presentata

25 Sui i precedenti del ciceroniano De oratore 2, 28, 159 (aurificis stadera vs popularis trutina) e di Galateo 8 (stadera del mugnaio vs bilancia dell'orafo) e l'opposizione retorica/logica che vi è simboleggiata, cf. Battistini (1989, 99; 2005, 100ss.) e Bellini (1997, 73).

26 Ricordo che scandaglio poteva significare anche il controllo delle merci dichiarate alla dogana (cf. GDLI).

27 Per le vicende e la descrizione del frontespizio del Saggiatore cf. quanto scrivono Besomi/ Helbing (2005, 437-440). Ciardi/Tongiorgi-Tomasi (1985) non trattano del Saggiatore, ma offrono un quadro generale sul rapporto titolazione/disegno nei frontespizi di testi scientifici secenteschi. Utile anche Pantin (2010). 
così di sfuggita, dovette essere cara a chi, nella Bilancetta, aveva (ri)costruito e descritto la bilancia idrostatica con cui Archimede avrebbe appurato il furto dell'oro nella corona di Gerone. La più generale, e felicissima, corrispondenza tra saggiatore e scienziato moderno fu raccolta da Bonaventura Cavalieri, che definì il Pisano come «l'esquisitissimo saggiatore della natura». ${ }^{28}$

\section{Bibliografia}

Le citazioni galileiane sono tratte dall'Edizione Nazionale (EN) a cura di A. Favaro (Firenze, Barbèra, 1890-1909), oggi disponibile anche on-line sul sito del Museo Galileo di Firenze. ${ }^{29}$

Altieri Biagi, Maria Luisa, Galileo e la terminologia tecnico-scientifica, Firenze, Olschki, 1965.

Battistini, Andrea, Introduzione a Galilei, Roma/Bari, Laterza, 1989.

Battistini, Andrea (ed.), Galileo Galilei, Sidereus nuncius, Venezia, Marsilio, 1993.

Battistini, Andrea, «Girandole» verbali e «severità di geometriche dimostrazioni». Battaglie linguistiche nel «Saggiatore», Galilaeana 2 (2005), 87-106.

Bellini, Eraldo, Umanisti e Lincei, Padova, Antenore, 1997.

Besomi, Ottavio/Helbing, Mario (edd.), Galileo Galilei, Il Saggiatore, Padova, Antenore, 2005.

Bisetto, Antonietta, La formazione delle parole, in: Salvi, Giampaolo/Renzi, Lorenzo (edd.), Grammatica dell'italiano antico, Bologna, il Mulino, 2010, cap. 41.

Buonarroti, Michelangelo, La Fiera commedia di Michelagnolo Buonarruoti il Giovane e la Tancia commedia rusticale del medesimo coll'annotazioni dell'abate Anton Maria Salvini [...], Firenze, Tartini e Franchi, 1726.

Cavalieri, Bonaventura, Lo specchio ustorio overo Trattato delle settioni coniche [...], Bologna, Dozza, 1632.

Chauviré, Christiane, L'Essayeur de Galilée, Paris, Les Belles Lettres, 1979.

Ciardi, Roberto Paolo/Tongiorgi-Tomasi, Lucia, La «scienza» illustrata: osservazioni sui frontespizi delle opere di Athanasius Kircher e di Galileo Galilei, Annali dell'Istituto storico italogermanico in Trento 11 (1985), 69-78.

28 Vale la pena riportare il passo per intero, giacché si coglie anche la valorizzazione della matematica: «Ma quanto vi aggiunga la cognitione delle scienze matematiche, giudicate da quelle famosissime scuole de' Pitagorici, e de' Platonici, sommamente necessarie per intender le cose fisiche, spero in breve sarà manifesto per la nuova dottrina del moto promessaci dall'esquisitissimo saggiatore della natura, dico dal sig. Galileo Galilei, ne' suoi Dialogi [...]» (Cavalieri 1632, 152). In un intervento del 1942 che ha di fatto fondato gli studi linguistici su Galileo ed è tuttora valido, Bruno Migliorini si avvicinava alla caratterizzazione fornita da Cavalieri, presentando così uno degli atteggiamenti mentali e linguistici dello scienziato: «Egli confronta la sua opera con quella d'un «saggiatore», che non con una libra grossolana, ma «con bilancia esquisita e giusta〉 pondera le cose e le sottopone a «cimento» (Migliorini 1948, 155).

29 <http://pinakes.imss.fi.it:8080/pinakestext/home.seam?conversationId=1246>. 
Crusca $=$ Vocabolario degli Accademici della Crusca, Venezia, Giovanni Alberti 1612; seconda ed.: Venezia, lacopo Sarzina, 1623; terza ed.: Firenze, Accademia della Crusca, 1691; quarta ed.: Firenze, Domenico Maria Manni, 1729-1738; quinta ed. (A-0): Firenze, Tipografia Galileana, 1863-1923.

d'Angelis, Antonella, La derivazione nominale e aggettivale in italiano e in spagnolo. La suffissazione, Roma, Aracne, 2006.

Dardano, Maurizio, La formazione delle parole nell'italiano di oggi, Roma, Bulzoni, 1978.

DELI = Cortelazzo, Manlio/Zolli, Paolo, DELI. Dizionario etimologico della lingua italiana, Bologna, Zanichelli, ${ }^{2} 1999$ ('1979-1988).

Del Lungo, Isidoro/Favaro, Antonio, La prosa di Galileo per saggi criticamente disposti, Firenze, Sansoni, 1911.

Dietz, Gunther, Titel wissenschaftlicher Texte, Tübingen, Narr, 1995.

Drake, Stillman (ed.), Discoveries and Opinions of Galileo, Garden City (NY), Doubleday, 1957.

Drake, Stillman, The Starry Messenger, Isis 49 (1958), 346s.

Drake, Stillman, Galileo at Work. His Scientific Biography, New York, Dover, 1995.

Drake, Stillman/O'Malley Charles D. (edd.), The Controversy on the Comets of 1618: Galileo Galilei, Horatio Grassi, Mario Guiducci, Johann Kepler [testi di Galilei, Grassi, Guiducci, Kepler tradotti dal latino e dall'italiano], University of Pennsylvania Press, Philadelphia 1960.

Favaro, Antonio, Saggio di una traduzione italiana del «Sidereus nuncius» per cura di Vincenzio Viviani, Atti e memorie della Reale Accademia di scienze, lettere ed arti in Padova 8 (1892), 34-41.

GDLI = Battaglia, Salvatore, Grande dizionario della lingua italiana, 21 vol., Torino, UTET, 1961-2002.

Genette, Gérard, Seuils, Paris, Seuil, 1987.

GRADIT = De Mauro, Tullio, Grande dizionario italiano dell'uso, 8 vol., Torino, UTET, 1999-2007.

Grassi, Orazio, Libra astronomica ac philosophica [...], Perusiae, Naccarini, 1619.

Griselini, Francesco/Fassadoni, Marco, Dizionario delle arti e de' mestieri, vol. 14, Venezia, Fenzo, 1773.

Hodierna, Giovanni Battista, Archimede redivivo [...], Palermo, Decio Cirillo, 1644.

Kepler, Johannes, Gesammelte Werke, vol. 8, ed. Franz Hammer, München, Beck, 1963.

$\mathrm{LEI}=$ Pfister, Max/Schweickard, Wolfgang (edd.), LEI. Lessico Etimologico Italiano, Wiesbaden, Reichert, 1979ss.

$\mathrm{LIZ}=\mathrm{LIZ}$. Letteratura Italiana Zanichelli. CD-ROM dei testi della letteratura italiana, Bologna, Zanichelli, ${ }^{4} 2001$.

Lo Duca, Maria G., «Nomi di agente» e «Nomi di strumento», in: Grossmann, Maria/Rainer, Franz, La formazione delle parole in italiano, Tübingen, Niemeyer, 2004, 351-374.

Lüdtke, Jens, Romanische Wortbildung. Inhaltlich, diachronisch, synchronisch, Tübingen, Stauffenburg, 2005.

Migliorini, Bruno, Galileo e la lingua italiana, in: id., Lingua e cultura, Roma, Tumminelli, 1948, 135-158.

Migliorini, Bruno, Storia della lingua italiana, Milano, Bompiani, 1994.

Nuovo dizionario universale tecnologico o di arti e mestieri e della economia industriale $e$ commerciante, prima traduzione italiana fatta da una società di dotti e di artisti, vol. 11, Venezia, Antonelli, 1833.

Pantin, Isabelle (ed.), Galileo Galilei, Sidereus nuncius. Le messager celeste, Paris, Les Belles Lettres, 1992. 
Pantin, Isabelle, Galilée, «metteur en texte»?, in: Riffaud, Alain (ed.), L'écrivain et l'imprimeur, Rennes, Presses Universitaires de Rennes, 2010, 147-164.

Passarotti, Marco, La semantica concettuale della morfologia. I derivati in - tore/-trice e in - ata/ - uta/-ita, Roma, Aracne, 2007.

Patruno, Barbara, I derivati agentivi italiani. Varietà, tipicità, specificità, in: Grossmann, Maria/ Thornton, Anna Maria (edd.), La formazione delle parole. Atti del XXXVII Congresso internazionale di studi della Società di linguistica italiana (L'Aquila, 25-27 settembre 2003), Roma, Bulzoni, 2005, 431-442.

Poggi Salani, Teresa, L'atteggiamento linguistico di Lorenzo Magalotti e il lessico dei «Saggi di naturali esperienze», Acme 24 (1961), 7-69.

Premoli, Palmiro, Vocabolario nomenclatore illustrato. Il tesoro della lingua italiana: spiega e suggerisce parole, sinonimi, frasi, 2 vol., Milano, Aldo Manuzio, 1909-1912.

Reusch, Franz Heinrich, Der Process Galilei's und die Jesuiten, Bonn, Weber, 1879.

Rigutini, Giuseppe/Fanfani, Pietro, Vocabolario italiano della lingua parlata, Firenze, Barbèra, ${ }^{2} 1893$.

Rosen, Edward, The Title of Galileo's «Sidereus Nuncius», Isis 41 (1950), 287ss.

Rosen, Edward, [Recensione a Drake (1957)], Journal of the History of Ideas 18 (1957), 439-448.

Rothe, Arnold, Der literarische Titel. Funktionen, Formen, Geschichte, Frankfurt am Main, Klostermann, 1986.

Russo, François, Note sur la traduction du titre de l'ouvrage de Galilée «Sidereus Nuncius», Revue d'histoire des sciences 20 (1967), 67ss.

Scalise, Sergio, Preliminari per lo studio di un affisso: «- tore» 0 «- ore»?, in: Benincà, Paola, et al. (edd.), Italiano e dialetti nel tempo. Saggi di grammatica per Giulio C. Lepschy, Roma, Bulzoni, 1996, 291-307.

Scalise, Sergio/Bisetto, Antonietta, La struttura delle parole, Bologna, il Mulino, 2008.

Stelluti, Giovanni Battista, Scandaglio sopra la Libra astronomica e filosofica di Lotario Sarsi [...], Terni, Guerrieri, 1622.

Stephanus, Robertus, Dictionarium, seu latinae linguae Thesaurus, Parisiis, ex officina Roberti Stephani, 1531.

Targioni Tozzetti, Giovanni, Notizie degli aggrandimenti delle scienze fisiche accaduti in Toscana [...], vol. 2/1, Firenze, Bouchard 1780.

Tekavčić, Pavao, Grammatica storica dell'italiano, vol. 3: Lessico, Bologna, il Mulino, ${ }^{2} 1980$.

$\mathrm{TLIO}=$ Beltrami, Pietro G. (ed.), Tesoro della lingua italiana delle origini, Firenze, 1998ss., <http://www.vocabolario.org>.

Tommaseo, Niccolò/Bellini, Bernardo, Dizionario della lingua italiana, Torino, UTET, 1861-1879.

Zanetti, Guid'Antonio, Nuova raccolta delle monete e zecche d'Italia, vol. 1, Bologna, dalla Volpe, 1775. 